\title{
NON-TANGENTIAL HALF LIGHTLIKE SUBMANIFOLDS OF SEMI-RIEMANNIAN MANIFOLDS WITH SEMI-SYMMETRIC NON-METRIC CONNECTIONS
}

\author{
Dae Ho Jin
}

\begin{abstract}
In this paper, we construct two types of non-tangential half lightlike submanifolds of a semi-Riemannian manifold admitting a semisymmetric non-metric connection. Our main result is to prove several characterization theorems for each types of such half lightlike submanifolds equipped with totally geodesic screen distributions.
\end{abstract}

\section{Introduction}

The theory of lightlike submanifolds is an important topic of research in differential geometry due to its application in mathematical physics, especially in the general relativity. The study of such notion was initiated by Duggal and Bejancu [4] and later studied by many authors (see recent results in two books $[6,7])$. The class of codimension two lightlike submanifolds $M$ of a semiRiemannian manifold $\bar{M}$ is compose of two classes, which are called the half lightlike and coisotropic submanifolds of $\bar{M}$, by virtue of the rank of its radical distribution $\operatorname{Rad}(T M)=T M \cap T M^{\perp}$, where $T M$ and $T M^{\perp}$ are the tangent and normal bundles of $M$, respectively [3, 5]. Half lightlike submanifold [11, 12] is a special case of the general $r$-lightlike submanifold [4] such that $r=1$, and its geometry is more general form than that of coisotrophic submanifold [8] or lightlike hypersurface $[13,15]$. Much of the geometry on half lightlike submanifolds will be immediately generalized in a formal way to arbitrary $r$-lightlike submanifolds. For this reason, we study only half lightlike submanifolds.

The notion of a semi-symmetric non-metric connection on a Riemannian manifold was introduced by Ageshe and Chafle [1]. Recently many authors have studied lightlike submanifolds of semi-Riemannian manifolds admitting semi-symmetric non-metric connections (see [10] [17]). Most of authors that wrote on lightlike submanifolds $M$ of semi-Riemannian manifolds $\bar{M}$ admitting semi-symmetric non-metric connections fail to treat with the case that the

Received April 19, 2013; Revised September 25, 2013.

2010 Mathematics Subject Classification. Primary 53C25, 53C40, 53C50.

Key words and phrases. half lightlike submanifold, semi-Riemannian manifold with a semi-symmetric non-metric connection, totally geodesic screen distribution. 
structure vector field $\zeta$ of $\bar{M}$ is not tangent to $M$, but studied only the case that $\zeta$ is tangent to $M$. In case that the structure vector field $\zeta$ of $\bar{M}$ is tangent to $M$, we say that $M$ is a tangential lightlike submanifold of $\bar{M}$. There is only a paper on non-tangential lightlike submanifolds of a semi-Riemannian manifold with a semi-symmetric non-metric connections, studied by Jin [15].

In this paper, we study non-tangential half lightlike submanifolds $M$ of a semi-Riemannian manifold $\bar{M}$ admitting a semi-symmetric non-metric connection. There are several different types of non-tangential half lightlike submanifolds according to the form of the structure vector field of $\bar{M}$. We study two types of them here, which are called the ascreen and transversal half lightlike submanifolds. We prove several classification theorems for each types of such half lightlike submanifolds $M$ endow with totally geodesic screen distributions.

\section{Semi-symmetric non-metric connection}

Let $(\bar{M}, \bar{g})$ be a semi-Riemannian manifold. A connection $\bar{\nabla}$ on $\bar{M}$ is called a semi-symmetric non-metric connection $[1]$ if $\bar{\nabla}$ and its torsion tensor $\bar{T}$ satisfy

$$
\begin{gathered}
\left(\bar{\nabla}_{X} \bar{g}\right)(Y, Z)=-\pi(Y) \bar{g}(X, Z)-\pi(Z) \bar{g}(X, Y), \\
\bar{T}(X, Y)=\pi(Y) X-\pi(X) Y,
\end{gathered}
$$

for any vector fields $X, Y$ and $Z$ on $\bar{M}$, where $\pi$ is a 1 -form associated with a non-vanishing vector field $\zeta$, which is called the structure vector field, by

$$
\pi(X)=\bar{g}(X, \zeta)
$$

A codimension 2 submanifold $(M, g)$ of a semi-Riemannian manifold $\bar{M}$ is called a half lightlike submanifold $[3,5]$ if its radical distribution $\operatorname{Rad}(T M)=$ $T M \cap T M^{\perp}$ is a subbundle of the tangent bundle $T M$ and the normal bundle $T M^{\perp}$ of rank 1 . Then there exist two non-degenerate complementary vector bundles $S(T M)$ and $S\left(T M^{\perp}\right)$ of $\operatorname{Rad}(T M)$ in $T M$ and $T M^{\perp}$ respectively, which are called the screen and co-screen distributions on $M$, such that

$$
T M=\operatorname{Rad}(T M) \oplus_{\text {orth }} S(T M), T M^{\perp}=\operatorname{Rad}(T M) \oplus_{\text {orth }} S\left(T M^{\perp}\right),
$$

where $\oplus_{\text {orth }}$ denotes the orthogonal direct sum. We denote such a half lightlike submanifold by $M=(M, g, S(T M))$. Denote by $F(M)$ the algebra of smooth functions on $M$ and by $\Gamma(E)$ the $F(M)$ module of smooth sections of a vector bundle $E$ over $M$. Choose $L \in \Gamma\left(S\left(T M^{\perp}\right)\right)$ as a spacelike unit vector field, i.e., $\bar{g}(L, L)=1$, without loss of generality. Consider the orthogonal complementary distribution $S(T M)^{\perp}$ to $S(T M)$ in $T \bar{M}$. Certainly $\operatorname{Rad}(T M)$ and $S\left(T M^{\perp}\right)$ are subbundles of $S(T M)^{\perp}$. As $S\left(T M^{\perp}\right)$ is non-degenerate, we have

$$
S(T M)^{\perp}=S\left(T M^{\perp}\right) \oplus_{\text {orth }} S\left(T M^{\perp}\right)^{\perp},
$$

where $S\left(T M^{\perp}\right)^{\perp}$ is the orthogonal complementary to $S\left(T M^{\perp}\right)$ in $S(T M)^{\perp}$.

For any null section $\xi$ of $\operatorname{Rad}(T M)$, there exists a uniquely defined lightlike vector bundle $\operatorname{lt}(T M)$ and a null vector field $N$ of $\operatorname{ltr}(T M)$ satisfying

$$
\bar{g}(\xi, N)=1, \bar{g}(N, N)=\bar{g}(N, X)=\bar{g}(N, L)=0, \forall X \in \Gamma(S(T M)) .
$$


We call $N, \operatorname{lt}(T M)$ and $\operatorname{tr}(T M)=S\left(T M^{\perp}\right) \oplus_{\text {orth }}$ ltr $(T M)$ the lightlike transversal vector field, lightlike transversal vector bundle and transversal vector bundle of $M$ with respect to $S(T M)$, respectively [4]. Then $T \bar{M}$ is decomposed as

$$
\begin{aligned}
T \bar{M} & =T M \oplus \operatorname{tr}(T M)=\{\operatorname{Rad}(T M) \oplus \operatorname{tr}(T M)\} \oplus_{\text {orth }} S(T M) \\
& =\{\operatorname{Rad}(T M) \oplus l \operatorname{tr}(T M)\} \oplus_{\text {orth }} S(T M) \oplus_{\text {orth }} S\left(T M^{\perp}\right) .
\end{aligned}
$$

From now and in the sequel, we take $X, Y, Z, W \in \Gamma(T M)$, unless otherwise specified. Let $P$ be the projection morphism of $T M$ on $S(T M)$. Then the local Gauss and Weingartan formulas of $M$ and $S(T M)$ are given respectively by

$$
\begin{aligned}
& \bar{\nabla}_{X} Y=\nabla_{X} Y+B(X, Y) N+D(X, Y) L \\
& \bar{\nabla}_{X} N=-A_{N} X+\tau(X) N+\rho(X) L \\
& \bar{\nabla}_{X} L=-A_{L} X+\phi(X) N \\
& \nabla_{X} P Y=\nabla_{X}^{*} P Y+C(X, P Y) \xi \\
& \nabla_{X} \xi=-A_{\xi}^{*} X-\sigma(X) \xi
\end{aligned}
$$

where $\nabla$ and $\nabla^{*}$ are induced linear connections on $T M$ and $S(T M)$ respectively, $B$ and $D$ are called the local lightlike and screen second fundamental forms of $M$ respectively, $C$ is called the local second fundamental form on $S(T M) . A_{N}, A_{\xi}^{*}$ and $A_{L}$ are linear operators on $T M$, which are called the shape operators, and $\tau, \rho, \phi$ and $\sigma$ are 1-forms on $T M$. We say that

$$
h(X, Y)=B(X, Y) N+D(X, Y) L
$$

is the global second fundamental form tensor of $M$.

Using (2.1), (2.2) and (2.6), we show that $B$ and $D$ are symmetric, and

$$
\begin{gathered}
\left(\nabla_{X} g\right)(Y, Z)=-\pi(Y) g(X, Z)-\pi(Z) g(X, Y) \\
+B(X, Y) \eta(Z)+B(X, Z) \eta(Y), \\
T(X, Y)=\pi(Y) X-\pi(X) Y,
\end{gathered}
$$

where $T$ is the torsion tensor with respect to $\nabla$ and $\eta$ is a 1-form such that

$$
\eta(X)=\bar{g}(X, N) \text {. }
$$

From the facts that $B(X, Y)=\bar{g}\left(\bar{\nabla}_{X} Y, \xi\right)$ and $D(X, Y)=\bar{g}\left(\bar{\nabla}_{X} Y, L\right)$, we know that $B$ and $D$ are independent of the choice of $S(T M)$ and satisfy

$$
B(X, \xi)=0, \quad D(X, \xi)=-\phi(X) .
$$

In the entire discussion of this article, we shall assume that the structure vector field $\zeta$ of $\bar{M}$ to be unit spacelike vector field. Let $a, b$ and $e$ be smooth functions defined by $a=\pi(N), b=\pi(\xi)$ and $e=\pi(L)$. Then the above three local second fundamental forms are related to their shape operators by

$$
\begin{aligned}
& g\left(A_{\xi}^{*} X, Y\right)=B(X, Y)-b g(X, Y), \quad \bar{g}\left(A_{\xi}^{*} X, N\right)=0 \\
& g\left(A_{L} X, Y\right)=D(X, Y)-e g(X, Y)+\phi(X) \eta(Y) \\
& \bar{g}\left(A_{L} X, N\right)=\rho(X)-e \eta(X)
\end{aligned}
$$




$$
\begin{aligned}
& g\left(A_{N} X, P Y\right)=C(X, P Y)-a g(X, P Y)-\eta(X) \pi(P Y), \\
& \bar{g}\left(A_{N} X, N\right)=-a \eta(X), \quad \sigma(X)=\tau(X)-b \eta(X) .
\end{aligned}
$$

It follows from (2.14) that $A_{\xi}^{*}$ is $\Gamma(S(T M))$-valued self-adjoint and satisfies

$$
A_{\xi}^{*} \xi=0 .
$$

Definition. A distribution $D$ on $M$ is called a parallel distribution if

$$
\nabla_{X} Y=0, \quad \forall X \in \Gamma(T M) \text { and } \forall Y \in \Gamma(D) .
$$

In case $X \in \Gamma(D)$, we say that $D$ is auto-parallel distribution on $M$.

Definition. (1) A screen distribution $S(T M)$ is called totally geodesic [5] in $M$ if $C=0$ on a coordinate neighborhood $\mathcal{U}$ in $M$.

(2) A half lightlike submanifold $M$ is called totally umbilical [5] if there exist a transversal vector field $\mathcal{H} \in \Gamma(\operatorname{tr}(T M))$ on $\mathcal{U}$ such that

$$
h(X, Y)=\mathcal{H} g(X, Y), \quad \forall X, Y \in \Gamma(T M) .
$$

In case $\mathcal{H}=0$ on $\mathcal{U}$, we say that $M$ is totally geodesic.

It is easy to see that $M$ is totally umbilical if and only if there exist smooth functions $\beta$ and $\delta$ on $\mathcal{U}$ such that $\mathcal{H}=\beta N+\delta L$ and

$$
B(X, Y)=\beta g(X, Y), \quad D(X, Y)=\delta g(X, Y), \quad \forall X, Y \in \Gamma(T M) .
$$

Remark 2.1. If $S(T M)$ is totally geodesic in $M$, then, from (2.9), (2.10) and (2.17), we show that $\operatorname{Rad}(T M)$ and $S(T M)$ are auto-parallel distributions of $M$ such that $M=\operatorname{Rad}(T M) \oplus_{\text {orth }} S(T M)$. Thus, by de Rham's decomposition theorem [2], $M$ is locally a product manifold $M=\mathcal{C} \times M^{*}$, where $\mathcal{C}$ is a null curve tangent to $\operatorname{Rad}(T M)$ and $M^{*}$ is a leaf of $S(T M)$.

In general, $S(T M)$ is not necessarily integrable. The following result gives equivalent conditions for the integrability of $S(T M)$ :

Theorem 2.2 ([16]). Let $M$ be a half lightlike submanifold of a semi-Riemannian manifold $\bar{M}$ admitting a semi-symmetric metric connection. Then the following assertions are equivalent:

(1) The screen distribution $S(T M)$ is an integrable distribution.

(2) $C$ is symmetric, i.e., $C(X, Y)=C(Y, X)$ for all $X, Y \in \Gamma(S(T M))$.

(3) The shape operator $A_{N}$ is self-adjoint with respect to $g$, i.e.,

$$
g\left(A_{N} X, Y\right)=g\left(X, A_{N} Y\right), \quad \forall X, Y \in \Gamma(S(T M)) .
$$

Definition. A vector field $X$ on a manifold $\bar{M}$ is said to be conformal Killing [16] if $\overline{\mathcal{L}}_{X} \bar{g}=-2 \delta \bar{g}$ for any smooth non-vanishing function $\delta$, where the symbol $\overline{\mathcal{L}}_{X}$ denotes the Lie derivative with respect to $X$, that is,

$$
\left(\overline{\mathcal{L}}_{X} \bar{g}\right)(Y, Z)=X(\bar{g}(Y, Z))-\bar{g}([X, Y], Z)-\bar{g}(Y,[X, Z]),
$$

for all $Y, Z \in \Gamma(T \bar{M})$. In particular, if $\delta=0$ ( $\delta$ is a non-zero constant), then $X$ is called a Killing (homothetic Killing) vector field. A distribution $\mathcal{G}$ on $\bar{M}$ 
is called conformal Killing (Killing or homothetic Killing) if each vector field belonging to $\mathcal{G}$ is a conformal Killing (Killing or homothetic Killing).

Theorem 2.3 ([16]). Let $M$ be a half lightlike submanifold of a semi-Riemannian manifold $\bar{M}$ admitting a semi-symmetric non-metric connection. If $S\left(T M^{\perp}\right)$ is conformal Killing, then there exists a smooth function $\delta$ such that

$$
D(X, Y)=\delta g(X, Y), \quad \forall X, Y \in \Gamma(T M) .
$$

Proof. For any $X, Y, Z \in \Gamma(T \bar{M})$, using (2.1) and (2.2), we have

$$
\left(\overline{\mathcal{L}}_{X} \bar{g}\right)(Y, Z)=\bar{g}\left(\bar{\nabla}_{Y} X, Z\right)+\bar{g}\left(Y, \bar{\nabla}_{Z} X\right)-2 \pi(X) \bar{g}(Y, Z) .
$$

If $S\left(T M^{\perp}\right)$ is a conformal Killing distribution, then, using (2.8) and (2.15), we have $\bar{g}\left(\bar{\nabla}_{X} L, Y\right)=-D(X, Y)+e g(X, Y)$. Thus we obtain

$$
\left(\overline{\mathcal{L}}_{L} \bar{g}\right)(X, Y)=-2 D(X, Y), \quad \forall X, Y \in \Gamma(T M) .
$$

Thus we have $D(X, Y)=\delta g(X, Y)$ for all $X, Y \in \Gamma(T M)$.

Denote by $\bar{R}, R$ and $R^{*}$ the curvature tensors of the semi-symmetric nonmetric connection $\bar{\nabla}$ on $\bar{M}$, the induced connection $\nabla$ on $M$ and the induced connection $\nabla^{*}$ on $S(T M)$ respectively. Using the Gauss-Weingarten formulas for $M$ and $S(T M)$, we obtain the Gauss-Codazzi equations for $M$ and $S(T M)$ :

$$
\begin{aligned}
\bar{R}(X, Y) Z= & R(X, Y) Z+B(X, Z) A_{N} Y-B(Y, Z) A_{N} X \\
& +D(X, Z) A_{L} Y-D(Y, Z) A_{L} X \\
& +\left\{\left(\nabla_{X} B\right)(Y, Z)-\left(\nabla_{Y} B\right)(X, Z)\right. \\
& +B(Y, Z)[\tau(X)-\pi(X)]-B(X, Z)[\tau(Y)-\pi(Y)] \\
& +D(Y, Z) \phi(X)-D(X, Z) \phi(Y)\} N \\
& +\left\{\left(\nabla_{X} D\right)(Y, Z)-\left(\nabla_{Y} D\right)(X, Z)+B(Y, Z) \rho(X)\right. \\
& -B(X, Z) \rho(Y)-D(Y, Z) \pi(X)+D(X, Z) \pi(Y)\} L,
\end{aligned}
$$

$$
\begin{aligned}
\bar{R}(X, Y) N= & -\nabla_{X}\left(A_{N} Y\right)+\nabla_{Y}\left(A_{N} X\right)+A_{N}[X, Y] \\
& +\tau(X) A_{N} Y-\tau(Y) A_{N} X+\rho(X) A_{L} Y-\rho(Y) A_{L} X \\
& +\left\{B\left(Y, A_{N} X\right)-B\left(X, A_{N} Y\right)+2 d \tau(X, Y)\right. \\
& +\phi(X) \rho(Y)-\phi(Y) \rho(X)\} N \\
& +\left\{D\left(Y, A_{N} X\right)-D\left(X, A_{N} Y\right)+2 d \rho(X, Y)\right. \\
& +\rho(X) \tau(Y)-\rho(Y) \tau(X)\} L,
\end{aligned}
$$

$$
\begin{aligned}
\bar{R}(X, Y) L= & -\nabla_{X}\left(A_{L} Y\right)+\nabla_{Y}\left(A_{L} X\right)+A_{L}[X, Y] \\
& +\phi(X) A_{N} Y-\phi(Y) A_{N} X \\
& +\left\{B\left(Y, A_{L} X\right)-B\left(X, A_{L} Y\right)+2 d \phi(X, Y)\right. \\
& +\tau(X) \phi(Y)-\tau(Y) \phi(X)\} N ;
\end{aligned}
$$




$$
\begin{aligned}
R(X, Y) P Z= & R^{*}(X, Y) P Z+C(X, P Z) A_{\xi}^{*} Y-C(Y, P Z) A_{\xi} X \\
& +\left\{\left(\nabla_{X} C\right)(Y, P Z)-\left(\nabla_{Y} C\right)(X, P Z)\right. \\
& +C(X, P Z)[\sigma(Y)+\pi(Y)]-C(Y, P Z)[\sigma(X)+\pi(X)]\} \xi
\end{aligned}
$$

$$
\begin{aligned}
R(X, Y) \xi= & -\nabla_{X}^{*}\left(A_{\xi}^{*} Y\right)+\nabla_{Y}^{*}\left(A_{\xi}^{*} X\right)+A_{\xi}^{*}[X, Y] \\
& +\sigma(Y) A_{\xi}^{*} X-\sigma(X) A_{\xi}^{*} Y \\
& +\left\{C\left(Y, A_{\xi}^{*} X\right)-C\left(X, A_{\xi}^{*} Y\right)-2 d \sigma(X, Y)\right\} \xi
\end{aligned}
$$

In case $R=0\left\{R^{*}=0\right\}$, we say that $M$ the leaf $M^{*}$ of $\left.S(T M)\right\}$ is flat.

Definition. A complete connected semi-Riemannian manifold $\bar{M}$ of constant curvature $c$ is called a semi-Riemannian space form, and denote it by $\bar{M}(c)$. For any vector fields $X, Y, Z$ of $\bar{M}$, the curvature tensor $\bar{R}$ of $\bar{M}(c)$ is given by

$$
\bar{R}(X, Y) Z=c\{\bar{g}(Y, Z) X-\bar{g}(X, Z) Y\} .
$$

Taking the scalar product with $\xi$ and $L$ to $(2.24)$, we get

$$
\bar{g}(\bar{R}(X, Y) Z, \xi)=0, \quad \bar{g}(\bar{R}(X, Y) Z, L)=0,
$$

for all $X, Y, Z \in \Gamma(T M)$. From this results and (2.17), we obtain

$$
\begin{aligned}
\bar{R}(X, Y) Z= & R(X, Y) Z+B(X, Z) A_{N} Y-B(Y, Z) A_{N} X \\
& +D(X, Z) A_{L} Y-D(Y, Z) A_{L} X, \quad \forall X, Y, Z \in \Gamma(T M) .
\end{aligned}
$$

\section{Ascreen half lightlike submanifolds}

Definition. A half lightlike submanifold $M$ of a semi-Riemannian manifold $\bar{M}$ admitting a semi-symmetric non-metric connection is called an ascreen half lightlike submanifold $[9,15]$ if $\zeta$ belongs to $\operatorname{Rad}(T M) \oplus \operatorname{ltr}(T M)$.

If $M$ is an ascreen half lightlike submanifold of $\bar{M}$, then $\zeta$ is expressed as

$$
\zeta=a \xi+b N, \quad e=0 .
$$

As $\bar{g}(\zeta, \zeta)=1$, we have $2 a b=1$. This implies that $a \neq 0$ and $b \neq 0$. Denote by $(2.16)_{i}$ the $i$-th equation of (2.16). We use same notations for any others. Taking the scaler product with $X$ to $(3.1)$, we have $\pi(X)=b \eta(X)$ for all $X \in \Gamma(T M)$. Comparing this equation with $(2.16)_{3}$, we obtain

$$
\tau(X)=\pi(X)+\sigma(X), \quad \forall X \in \Gamma(T M) .
$$

Theorem 3.1. Let $M$ be an ascreen half lightlike submanifold of a semiRiemannian manifold $\bar{M}$ admitting a semi-symmetric non-metric connection. Then the screen distribution $S(T M)$ is an integrable distribution.

Proof. Taking the scalar product with $\xi$ to (2.20) and $N$ to (2.19) such that $Z=\xi$ by turns and using (2.13), $(2.15)_{2}$ and (2.23), we obtain

$$
\begin{aligned}
& \bar{g}(\bar{R}(X, Y) \xi, N) \\
= & B\left(X, A_{N} Y\right)-B\left(Y, A_{N} X\right)-2 d \tau(X, Y)+\rho(X) \phi(Y)-\rho(Y) \phi(X)
\end{aligned}
$$




$$
=C\left(Y, A_{\xi}^{*} X\right)-C\left(X, A_{\xi}^{*} Y\right)-2 d \sigma(X, Y)+\rho(X) \phi(Y)-\rho(Y) \phi(X) .
$$

From the above two representations, we deduces to the following equation:

$$
\begin{aligned}
& B\left(X, A_{N} Y\right)-B\left(Y, A_{N} X\right)-2 d \tau(X, Y) \\
= & C\left(Y, A_{\xi}^{*} X\right)-C\left(X, A_{\xi}^{*} Y\right)-2 d \sigma(X, Y) .
\end{aligned}
$$

Substituting (2.14) and (2.16) into the last equation and using (3.2) and the facts that $\pi\left(A_{\xi}^{*} X\right)=0$ for any $X \in \Gamma(T M)$ and $A_{\xi}^{*}$ is self-adjoint, we have

$$
2 d \pi(X, Y)=b\left\{g\left(X, A_{N} Y\right)-g\left(A_{N} X, Y\right)\right\}, \quad \forall X, Y \in \Gamma(T M) .
$$

As $\pi=0$ on $S(T M)$, we show that $d \pi=0$ on $S(T M)$. Thus we obtain

$$
g\left(A_{N} X, Y\right)=g\left(X, A_{N} Y\right), \quad \forall X, Y \in \Gamma(S(T M)) .
$$

It follows from Theorem 2.2 that $S(T M)$ is an integrable distribution.

Theorem 3.2. Let $M$ be an ascreen half lightlike submanifold of a semiRiemannian space form $\bar{M}(c)$ admitting a semi-symmetric non-metric connection. If $S(T M)$ is totally geodesic and $S\left(T M^{\perp}\right)$ is conformal Killing, then

(1) $c=0$,

(2) $M$ and $M^{*}$ are totally geodesic and flat manifolds,

(3) $S\left(T M^{\perp}\right)$ is a Killing and parallel distribution, and

(4) $M$ is locally a product flat manifold $\mathcal{C} \times M^{*}$, where $\mathcal{C}$ is a null curve tangent to $\operatorname{Rad}(T M)$ and $M^{*}$ is a flat leaf of the integrable $S(T M)$.

Proof. Using (2.16) and the fact that $\pi(P X)=0$, we show that $S(T M)$ is totally geodesic in $M$ if and only if the shape operator $A_{N}$ satisfies

$$
A_{N} X=-a X, \quad \forall X \in \Gamma(T M) .
$$

Taking the scalar product with $N$ to (2.22) and using $C=0$, we get

$$
\bar{g}(R(X, Y) P Z, N)=0, \quad \forall X, Y, Z \in \Gamma(T M) .
$$

Taking the scalar product with $N$ to (2.26) such that $Z=P Z$ and using $(2.15)_{2},(2.16)_{2},(2.24),(2.18)$ and the last equation, we obtain

$$
\begin{aligned}
& \{c \eta(X)+\delta \rho(X)\} g(Y, P Z)-\{c \eta(Y)+\delta \rho(Y)\} g(X, P Z) \\
= & a\{B(Y, P Z) \eta(X)-B(X, P Z) \eta(Y)\} .
\end{aligned}
$$

Replacing $X$ by $\xi$ to this equation and using $(2.13)_{1}$, we have

$$
(c+\delta \rho(\xi)) g(X, Y)=a B(X, Y), \quad \forall X, Y \in \Gamma(T M) .
$$

As $a \neq 0$, let $\beta=a^{-1}(c+\delta \rho(\xi))$. Then we obtain

$$
B(X, Y)=\beta g(X, Y), \quad \forall X, Y \in \Gamma(T M) .
$$

From (2.18) and (3.4), we show that $M$ is totally umbilical.

As $S\left(T M^{\perp}\right)$ is conformal Killing, from (2.15), (2.18) and the facts that $\phi=e=0$ and $X=P X+\eta(X) \xi$, we have

$$
A_{L} X=\delta X+(\rho(X)-\delta \eta(X)) \xi,
$$


for all $X \in \Gamma(T M)$. Substituting (2.24) and (3.5) into (2.21) and using (2.13) and the facts that $\phi=0$ and $B$ is symmetric, we have

$$
\nabla_{X}\left(A_{L} Y\right)-\nabla_{Y}\left(A_{L} X\right)-A_{L}[X, Y]=0,
$$

for all $X, Y \in \Gamma(T M)$. Applying $\nabla_{Y}$ to (3.5) and using (2.10), we have

$$
\begin{aligned}
\nabla_{X}\left(A_{L} Y\right)= & X[\delta] Y+\delta \nabla_{X} Y-(\rho(Y)-\delta \eta(Y)) A_{\xi}^{*} X \\
& +\{X[\rho(Y)-\delta \eta(Y)]-\sigma(X)(\rho(Y)-\delta \eta(Y))\} \xi .
\end{aligned}
$$

Substituting this equation into (3.6) and using (2.12) and (3.5), we have

$$
\begin{aligned}
& \{X[\delta]-\delta \pi(X)\} Y-\{Y[\delta]-\delta \pi(Y)\} X \\
& +(\rho(X)-\delta \eta(X)) A_{\xi}^{*} Y-(\rho(Y)-\delta \eta(Y)) A_{\xi}^{*} X \\
& +\{2 d \rho(X, Y)-2 \delta d \eta(X, Y)-X[\delta] \eta(Y)+Y[\delta] \eta(X) \\
& -\sigma(X)(\rho(Y)-\delta \eta(Y))+\sigma(Y)(\rho(X)-\delta \eta(X))\} \xi=0 .
\end{aligned}
$$

Taking the scalar product with $N$ to (3.7) and using (2.14) 2 and (3.2), we have

$$
\begin{aligned}
& 2 d \rho(X, Y)-2 \delta d \eta(X, Y)+\delta \tau(X) \eta(Y)-\delta \tau(Y) \eta(X) \\
& +2 \delta\{\pi(Y) \eta(X)-\pi(X) \eta(Y)\}+\rho(X) \sigma(Y)-\rho(Y) \sigma(X)=0 .
\end{aligned}
$$

Applying $\bar{\nabla}_{X}$ to $\eta(Y)=\bar{g}(Y, N)$ and using (2.1), (2.6), (2.7) and (3.3), we get

$$
X(\eta(Y))=-\pi(Y) \eta(X)+\eta\left(\nabla_{X} Y\right)+\tau(X) \eta(Y) \text {. }
$$

Substituting this relation into $2 d \eta(X, Y)=X(\eta(Y))-Y(\eta(X))-\eta([X, Y])$ and using (2.12) and (3.3), we have

$$
2 d \eta(X, Y)=\tau(X) \eta(Y)-\tau(Y) \eta(X) .
$$

Substituting this into (3.8) and using the fact that $\pi(X)=b \eta(X)$, we have

$$
2 d \rho(X, Y)+\rho(X) \sigma(Y)-\rho(Y) \sigma(X)=0 .
$$

On the other hand, taking the scalar product with $L$ to (2.20) and using (2.24), (3.3) and the fact that $D$ is symmetric, we get

$$
2 d \rho(X, Y)+\rho(X) \tau(Y)-\rho(Y) \tau(X)=0 .
$$

Subtracting the last two equations and then, using (3.2) we obtain

$$
\rho(X) \pi(Y)=\rho(Y) \pi(X), \quad \forall X, Y \in \Gamma(T M) .
$$

Replacing $Y$ by $\xi$ to this and using $\pi(X)=b \eta(X)$ and $b \neq 0$, we have

$$
\rho(X)=\rho(\xi) \eta(X), \quad \forall X \in \Gamma(T M) .
$$

Taking the scalar product with $P Z$ to $(3.7)$ and using $(2.14)_{1}$, we have

$$
\begin{aligned}
& \{X[\delta]-\delta \pi(X)+(\beta-b)(\rho(X)-\delta \eta(X))\} g(Y, Z) \\
= & \{Y[\delta]-\delta \pi(Y)+(\beta-b)(\rho(Y)-\delta \eta(Y))\} g(X, Z) .
\end{aligned}
$$

Replacing $X$ by $\xi$ and $Z$ by $Y$ to this such that $g(Y, Y) \neq 0$, we obtain

$$
\xi[\delta]-\delta \beta+(\beta-b) \rho(\xi)=0 .
$$


Taking the scalar product with $L$ to $(2.19)$ and using (2.24), we have

$$
\begin{aligned}
& \left(\nabla_{X} D\right)(Y, Z)-\left(\nabla_{Y} D\right)(X, Z) \\
= & B(X, Z) \rho(Y)-B(Y, Z) \rho(X)-D(X, Z) \pi(Y)+D(Y, Z) \pi(X) .
\end{aligned}
$$

Applying $\nabla_{Z}$ to $(2.18)$ and using $(2.11),(3.4)$ and $\pi(X)=b \eta(X)$, we get

$$
\left(\nabla_{X} D\right)(Y, Z)=X[\delta] g(Y, Z)+\delta(\beta-b)\{g(X, Z) \eta(Y)-g(X, Y) \eta(Z)\} .
$$

Substituting this, (2.18), (3.4) and $\pi(X)=b \eta(X)$ into (3.11), we have

$$
\begin{aligned}
& \{X[\delta]-\delta \beta \eta(X)+\beta \rho(X)\} g(Y, Z) \\
= & \{Y[\delta]-\delta \beta \eta(Y)+\beta \rho(Y)\} g(X, Z) .
\end{aligned}
$$

Taking $X=\xi$ and $Y=Z$ to this equation such that $g(Y, Y) \neq 0$, we obtain

$$
\xi[\delta]-\delta \beta+\beta \rho(\xi)=0 .
$$

Subtracting (3.13) from (3.10) and using the fact that $b \neq 0$, we obtain $\rho(\xi)=0$.

It follows from (3.9) that $\rho=0$. In this case, we show that $\beta=c / a$.

Taking the scalar product with $P Z$ to $(2.20)$ and using $\rho=0$, we have

$$
\begin{aligned}
\bar{g}(\bar{R}(X, Y) N, P Z)= & g\left(-\nabla_{X}\left(A_{N} Y\right)+\nabla_{Y}\left(A_{N} X\right)+A_{N}[X, Y], P Z\right) \\
& +\tau(X) g\left(A_{N} Y, P Z\right)-\tau(Y) g\left(A_{N} X, P Z\right)
\end{aligned}
$$

for any $X, Y, Z \in \Gamma(T M)$. Applying $\nabla_{X}$ to $A_{N} Y=-a Y$, we have

$$
\nabla_{X}\left(A_{N} Y\right)=-X[a] Y-a \nabla_{X} Y \text {. }
$$

Substituting this, (2.24) and (3.3) into (3.14), we have

$$
\begin{aligned}
& \{X[a]-a \pi(X)-a \tau(X)+c \eta(X)\} g(Y, Z) \\
= & \{Y[a]-a \pi(Y)-a \tau(Y)+c \eta(Y)\} g(X, Z) .
\end{aligned}
$$

Taking $X=\xi$ and $Y=Z$ to this such that $g(Y, Y) \neq 0$, we obtain

$$
\xi[a]-a b-a \tau(\xi)+c=0 .
$$

Taking the scalar product with $\xi$ to $(2.19)$ and using $(2.25)_{1}$, we have

$$
\begin{aligned}
& \left(\nabla_{X} B\right)(Y, Z)-\left(\nabla_{Y} B\right)(X, Z) \\
= & \{\pi(X)-\tau(X)\} B(Y, Z)-\{\pi(Y)-\tau(Y)\} B(X, Z) .
\end{aligned}
$$

Applying $\nabla_{X}$ to $B(Y, Z)=\beta g(Y, Z)$ and using (2.11) and (3.4), we have

$$
\begin{aligned}
\left(\nabla_{X} B\right)(Y, Z)= & X[\beta] g(Y, Z)-\left\{\beta \pi(Y)-\beta^{2} \eta(Y)\right\} g(X, Z) \\
& -\left\{\beta \pi(Z)-\beta^{2} \eta(Z)\right\} g(X, Y) .
\end{aligned}
$$

Substituting this equation into (3.16), we get

$$
\left\{X[\beta]+\beta \tau(X)-\beta^{2} \eta(X)\right\} g(Y, Z)=\left\{Y[\beta]+\beta \tau(Y)-\beta^{2} \eta(Y)\right\} g(X, Z) .
$$

Taking $X=\xi$ and $Y=Z$ to this such that $g(Y, Y) \neq 0$, we obtain

$$
\xi[\beta]+\beta \tau(\xi)-\beta^{2}=0 .
$$


Replacing $\beta$ by $c / a$ to this equation and using the facts that $a \neq 0$, we have

$$
c\{\xi[a]-a \tau(\xi)+c\}=0 .
$$

Now we assume that $c \neq 0$. Then the last equation is reduced to

$$
\xi[a]-a \tau(\xi)+c=0 .
$$

Comparing (3.15) with (3.17), we have $a b=0$. It is a contradiction to $a b \neq 0$.

It follows that $c=0$, i.e., $\bar{R}=0$. As $c=0$, we get $\beta=B=0$.

Substituting (2.18) and (3.5) into (2.26) and using $\bar{R}=B=0$, we have

$$
\begin{aligned}
R(X, Y) Z & =\delta^{2}\{g(Y, Z) X-g(X, Z) Y\} \\
& -\delta^{2}\{g(Y, Z) \eta(X)-g(X, Z) \eta(Y)\} \xi .
\end{aligned}
$$

As $C=0$, from $(2.22)$ we see that $R(X, Y) P Z=R^{*}(X, Y) P Z$ for all $X, Y, Z \in$ $\Gamma(T M)$. This implies $R(X, Y) P Z \in \Gamma(S(T M))$. Thus we obtain

$$
\begin{gathered}
R^{*}(X, Y) P Z=\delta^{2}\{g(Y, P Z) X-g(X, P Z) Y\}, \\
\delta^{2}\{g(Y, P Z) \eta(X)-g(X, P Z) \eta(Y)\}=0,
\end{gathered}
$$

for all $X, Y, Z \in \Gamma(T M)$. Taking $X=\xi$ and $Y=P Z$ to (3.20) such that $g(P Z, P Z) \neq 0$, we have $\delta=0$. It follows from (3.18) and (3.19) that $M$ and $M^{*}$ are flat manifolds. As $\beta=\delta=0, M$ is totally geodesic and $S\left(T M^{\perp}\right)$ is a Killing distribution by (2.18) and (3.4). As $\rho=\delta=0$, we see that $A_{L}=0$ by (3.5). It follows from (2.8) that $\bar{\nabla}_{X} L=0$. Thus $S\left(T M^{\perp}\right)$ is a parallel distribution. From these results and Remark 2.1 we have our theorem.

\section{Transversal half lightlike submanifolds}

Definition. A half lightlike submanifold $M$ of a semi-Riemannian manifold $\bar{M}$ admitting a semi-symmetric non-metric connection is called a transversal half lightlike submanifold [9] if $\zeta$ belongs to the transversal vector bundle $\operatorname{tr}(T M)$.

If $M$ is a transversal half lightlike submanifold of $\bar{M}$, then $\zeta$ is decomposed as $\zeta=e L+b N$. Since $1=\bar{g}(\zeta, \zeta)=e^{2} \bar{g}(L, L)=e^{2}$, i.e., $e= \pm 1$, we may assume that $e=1$, without loss of generality. In this case, we have

$$
\zeta=L+b N, \quad a=0 .
$$

In this section, by saying that transversal half lightlike submanifolds we shall mean half lightlike submanifolds satisfying (4.1) such that $b \neq 0$.

Taking the scaler product with $X$ to $(4.1)$, we have $\pi(X)=b \eta(X)$ for all $X \in \Gamma(T M)$. Comparing this equation with $(2.16)_{3}$, we obtain

$$
\tau(X)=\pi(X)+\sigma(X), \quad \forall X \in \Gamma(T M) .
$$

Theorem 4.1. Let $M$ be a transversal half lightlike submanifold of a semiRiemannian manifold $\bar{M}$ admitting a semi-symmetric non-metric connection. Then the screen distribution $S(T M)$ is an integrable distribution. 
Proof. By using the method of Theorem 3.1, we obtain

$$
\begin{aligned}
& B\left(X, A_{N} Y\right)-B\left(Y, A_{N} X\right)-2 d \pi(X, Y) \\
= & C\left(Y, A_{\xi}^{*} X\right)-C\left(X, A_{\xi}^{*} Y\right)+\phi(X) \eta(Y)-\phi(Y) \eta(X) .
\end{aligned}
$$

Substituting (2.14) and (2.16) into the last equation and using the facts that $A_{\xi}^{*}$ is self-adjoint and $\pi\left(A_{\xi}^{*} X\right)=0$ for any $X \in \Gamma(T M)$, we have

$$
2 d \pi(X, Y)=b\left\{g\left(X, A_{N} Y\right)-g\left(A_{N} X, Y\right)\right\}+\eta(X) \phi(Y)-\eta(Y) \phi(X) .
$$

As $\pi=0$ on $S(T M)$, we show that $d \pi=0$ on $S(T M)$. Thus we obtain

$$
g\left(A_{N} X, Y\right)=g\left(X, A_{N} Y\right), \quad \forall X, Y \in \Gamma(S(T M)) .
$$

It follows from Theorem 2.2 that $S(T M)$ is an integrable distribution.

Theorem 4.2. Let $M$ be a transversal half lightlike submanifold of a semiRiemannian space form $\bar{M}(c)$ admitting a semi-symmetric non-metric connection. If $S(T M)$ is totally geodesic and $S\left(T M^{\perp}\right)$ is conformal Killing, then

(1) $c=0$,

(2) $M$ and $M^{*}$ are flat manifolds,

(3) $S\left(T M^{\perp}\right)$ is a homothetic Killing and parallel distribution, and

(4) $M$ is locally a product manifold $\mathcal{C} \times M^{*}$, where $\mathcal{C}$ is a null curve tangent to $\operatorname{Rad}(T M)$ and $M^{*}$ is a flat leaf of the integrable $S(T M)$.

Proof. Using (2.16) and the facts that $a=0$ and $\pi(P X)=0$, we show that the screen distribution $S(T M)$ is totally geodesic in $M$ if and only if $A_{N}=0$.

As $S\left(T M^{\perp}\right)$ is conformal Killing, from (2.15) and (2.18), we have

$$
A_{L} X=(\delta-1) X+(\rho(X)-\delta \eta(X)) \xi, \quad \forall X \in \Gamma(T M) .
$$

Taking the scalar product with $N$ to $(2.22)$ and using $C=0$, we get

$$
\bar{g}(R(X, Y) P Z, N)=0, \quad \forall X, Y, Z \in \Gamma(T M) .
$$

Taking the scalar product with $N$ to $(2.26)$ such that $Z=P Z$ and using the last equation, $(2.15)_{2},(2.24),(2.18)$ and the fact that $A_{N}=0$, we get

$$
\begin{aligned}
& \{c \eta(X)+\delta(\rho(X)-\eta(X))\} g(Y, Z) \\
= & \{c \eta(Y)+\delta(\rho(Y)-\eta(Y))\} g(X, Z) .
\end{aligned}
$$

Taking $X=\xi$ and $Y=Z$ to this equation such that $g(Y, Y) \neq 0$, we have

$$
c+\delta \rho(\xi)=\delta .
$$

Taking the scalar product with $P Z$ to $(2.20)$ and using $A_{N}=0$, we have

$$
\bar{g}(\bar{R}(X, Y) N, P Z)=\rho(X) g\left(A_{L} Y, P Z\right)-\rho(Y) g\left(A_{L} X, P Z\right) .
$$

Substituting (4.3) and (2.24) to this equation, we have

$$
\{c \eta(Y)+(\delta-1) \rho(Y)\} g(X, Z)=\{c \eta(X)+(\delta-1) \rho(X)\} g(Y, Z) .
$$

Taking $X=\xi$ and $Y=Z$ to this equation such that $g(Y, Y) \neq 0$, we have

$$
c+\delta \rho(\xi)=\rho(\xi) \text {. }
$$


Comparing (4.4) and (4.5), we obtain $\delta=\rho(\xi)$ and $c=\delta(1-\delta)$.

Taking the scalar product with $N$ to $(2.20)$ and using $(2.15)_{2}$ and the facts that $A_{N}=0$ and $\bar{g}(\bar{R}(X, Y) N, N)=0$, we have

$$
\rho(X) \eta(Y)=\rho(Y) \eta(X), \quad \forall X, Y \in \Gamma(T M) .
$$

Replacing $Y$ by $\xi$ to this equation, we have $\rho(X)=\rho(\xi) \eta(X)$ for any $X \in$ $\Gamma(T M)$. From this, the fact that $\delta=\rho(\xi)$ and (4.3), we obtain

$$
\rho(X)-\delta \eta(X)=0, \quad A_{L} X=(\delta-1) X, \quad \forall X \in \Gamma(T M) .
$$

Taking the scalar product with $L$ to $(2.19)$ and using $(2.25)_{2}$, we have

$$
\begin{aligned}
& \left(\nabla_{X} D\right)(Y, Z)-\left(\nabla_{Y} D\right)(X, Z) \\
= & B(X, Z) \rho(Y)-B(Y, Z) \rho(X)-D(X, Z) \pi(Y)+D(Y, Z) \pi(X) .
\end{aligned}
$$

Applying $\nabla_{X}$ to $D(Y, Z)=\delta g(Y, Z)$ and using (2.11), we have

$$
\begin{aligned}
\left(\nabla_{X} D\right)(Y, Z)= & X[\delta] g(Y, Z)-\delta \pi(Y) g(X, Z)-\delta \pi(Z) g(X, Y) \\
& +\delta B(X, Z) \eta(Y)+\delta B(X, Y) \eta(Z) .
\end{aligned}
$$

Substituting this equation into (4.7) and using (4.6) $)_{1}$, we get

$$
X[\delta] g(Y, Z)=Y[\delta] g(X, Z), \quad \forall X, Y \in \Gamma(T M) .
$$

Taking $X=\xi$ and $Y=Z$ to this such that $g(Y, Y) \neq 0$, we have $\xi[\delta]=0$.

Taking the scalar product with $P Z$ to $(2.21)$ and using $\phi=0$, we have

$$
\bar{g}(\bar{R}(X, Y) L, P Z)=g\left(-\nabla_{X}\left(A_{L} Y\right)+\nabla_{Y}\left(A_{L} X\right)+A_{L}[X, Y], P Z\right) .
$$

Applying $\nabla_{X}$ to $A_{L} Y=(\delta-1) Y$, we have

$$
\nabla_{X}\left(A_{L} Y\right)=X[\delta] Y+(\delta-1) \nabla_{X} Y
$$

Substituting this equation and (2.24) into the last equation, we have

$$
\{X[\delta]-(\delta-1) \pi(X)\} g(Y, Z)=\{Y[\delta]-(\delta-1) \pi(Y)\} g(X, Z) .
$$

Taking $X=\xi$ and $Y=Z$ to this equation such that $g(Y, Y) \neq 0$ and using the facts that $\xi[\delta]=0$ and $b \neq 0$, we obtain $\delta=1$. Substituting $\delta=\rho(\xi)=1$ into (4.4), we have $c=0$. As $\delta=1$, from $(4.6)_{2}$ we get $A_{L}=0$. From (2.26) and the facts that $A_{N}=0$ and $A_{L}=0$, we show that

$$
R(X, Y) Z=0, \quad \forall X, Y, Z \in \Gamma(T M) .
$$

Thus $M$ is flat. From this, $(2.22)$ and the fact that $C=0$, we also have

$$
R^{*}(X, Y) Z=0, \quad \forall X, Y, Z \in \Gamma(S(T M)) .
$$

Thus the leaf $M^{*}$ of $S(T M)$ is also flat. As $A_{L}=\phi=0$, from (2.8) we show that $S\left(T M^{\perp}\right)$ is a parallel distribution. As $\delta=1, S\left(T M^{\perp}\right)$ is homothetic Killing. From these results and Remark 2.1, we have our theorem. 


\section{References}

[1] N. S. Ageshe and M. R. Chafle, A semi-symmetric non-metric connection on a Riemannian manifold, Indian J. Pure Appl. Math. 23 (1992), no. 6, 399-409.

[2] G. de Rham, Sur la réductibilité d'un espace de Riemannian, Comm. Math. Helv. 26 (1952), 328-344.

[3] K. L. Duggal and A. Bejancu, Lightlike submanifolds of codimension 2, Math. J. Toyama Univ. 15 (1992), 59-82.

[4] — Lightlike Submanifolds of Semi-Riemannian Manifolds and Applications, Kluwer Acad. Publishers, Dordrecht, 1996.

[5] K. L. Duggal and D. H. Jin, Half-lightlike submanifolds of codimension 2, Math. J. Toyama Univ. 22 (1999), 121-161.

[6] _ Null curves and Hypersurfaces of Semi-Riemannian Manifolds, World Scientific, 2007.

[7] K. L. Duggal and B. Sahin, Differential geometry of lightlike submanifolds, Frontiers in Mathematics, Birkhäuser, 2010.

[8] D. H. Jin, Coistropic submanifolds of codimension 2, J. of Dongguk Univ. 19 (2000), $163-191$.

[9] _ Special half lightlike submanifolds of an indefinite cosymplectic manifold, Journal of Function Spaces and Applications 2012 (2012), Article ID 636242, 16 pages.

[10] L Lightlike submanifolds of a semi-Riemannian manifold with a semi-symmetric non-metric connection, J. Korean Soc. Math. Edu. Ser. B: Pure Appl. Math. 19 (2012), no. 3, 211-228.

[11] Einstein half lightlike submanifolds of a Lorentzian space form with a semisymmetric non-metric connection, J. Inequal. Appl. 2013 (2013), 403, 13 pp.

[12] Two characterization theorems for irrotational lightlike geometry, Commun. Korean Math. Soc. 28 (2013), no. 4, 809-818.

[13] Einstein lightlike hypersurfaces of a Lorentz space form with a semi-symmetric non-metric connection, Bull. Korean Math. Soc. 50 (2013), no. 4, 1367-1376.

[14] L Lightlike submanifolds of a semi-Riemannian manifold with a semi-symmetric non-metric connection, submitted in Taiwanese J. of Math.

[15] _ Ascreen lightlike hypersurfaces of a semi-Riemannian space form with a semisymmetric non-metric connection, accepted in Commun. Korean Math. Soc. 2013.

[16] D. H. Jin and J. W. Lee, A classification of half lightlike submanifolds of a semiRiemannian manifold with a semi-symmetric non-metric connection, Bull. Korean Math. Soc. 50 (2013), no. 3, 705-717.

[17] E. Yasar, A. C. Cöken, and A. Yücesan, Lightlike hypersurfaces in semi-Riemannian manifold with semi-symmetric non-metric connection, Math. Scand. 102 (2008), no. 2, $253-264$.

Department of Mathematics

DONGGUK UNIVERSITY

Gyeonguu 780-714, KoreA

E-mail address: jindh@dongguk.ac.kr 